\title{
El PODER EN LA PAZ IMPERFECTA Y EN FouCAULT ${ }^{\star}$
}

Cássia M. Rosato ${ }^{\star}$

\section{RESUMEN}

Este trabajo debate el concepto de poder en la paz imperfecta y en la obra de Foucault. El objetivo principal es demostrar que la perspectiva foucaultiana del poder no está lejos de la noción de poder en la paz imperfecta propuesta por Muñoz. Para tanto, ese artículo empieza con la idea de paz de UNESCO $y$ el contexto sociopolítico que favoreció el surgimiento de esa concepción. Enseguida, presenta características de la paz imperfecta, así como el concepto de poder está inserido en esta proposición y en Foucault. Al final, apunta tres semejanzas y/o aproximaciones existentes entre ambas las orientaciones que confirman su cercanía.

Palabras-clave: conflictos; Michel Foucault; paz imperfecta; poder.

\section{The POWer IN The IMPERfect PeACE AND IN Foucault}

\begin{abstract}
This article discuss the concept of power in the imperfect peace and in the work of Foucault. The main goal is demonstrate that Foucault's idea of power is not distant from the notion of power in the imperfect peace proposed by Muñoz. Therefore, this paper begins with the idea of peace from UNESCO and the sociopolitical context that encouraged the creation of this concept. Afterwards, presents the characteristics of the imperfect peace and how the idea of power is inserted in this proposition and in the work of Foucault. Finally, it's presented three similarities and/or proximities between both orientations that confirm its closeness.
\end{abstract}

Keywords: conflicts; Michel Foucault; imperfect peace; power.

\footnotetext{
^ Este trabajo tuvo el financiamiento de la Comisión Europea, a través del Programa Erasmus Mundus.

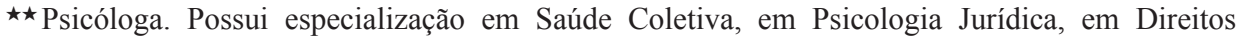
Humanos e é mestra em Psicologia pela Universidade Federal de Pernambuco (2011). Atualmente é mestranda do Instituto de Paz e Conflitos da Universidade de Granada (Espanha), pesquisadora do Projeto Itineris (MJ/ICMPD) e consultora da UNESCO.

E-mail: cassiarosato@yahoo.com.br
} 


\section{Paz y Conflictos}

La idea de paz y, sobre todo la paz como derecho humano de todos y algo imprescindible para la humanidad, fue propuesta por la Organización de las Naciones Unidas para la Educación, la Ciencia y la Cultura (UNESCO), en su Declaración de 1997. Además, en ese documento fue planteado el concepto de cultura de paz como importante substitución a una cultura de guerra. Sin embargo, se sabe que ese cambio no es sencillo y tampoco rápido; se trata de un cambio de cultura y eso lleva generaciones. Al mismo tiempo, esa Declaración es muy objetiva cuando define lo que son los derechos humanos, e incluye la paz como uno de ellos.

Ninguna nación, institución o persona debe sentirse autorizada a poseer y representar los derechos humanos ni menos aún a otorgar credenciales a los demás. Los derechos humanos no se tienen ni se ofrecen, sino que se conquistan y se merecen cada día. Tampoco deben considerarse una abstracción, sino pautas concretas de acción que deben incorporarse a la vida de todos los hombres y las mujeres, y a las leyes de cada país (UNESCO, 1997, p. 5).

Eso significa que para tener la paz y también los otros derechos humanos es necesario luchar ya que no es algo dado, o sea, las personas, instituciones y naciones necesitan conquistar sus derechos y eso ocurre de modo concreto y no como una abstracción.

Esa Declaración fue complementada por otra de las Naciones Unidas (ONU), en 1999, llamada Declaración y Programa de Acción sobre una Cultura de Paz. En ese momento, la expresión "cultura de paz" gana fuerza política, desde el punto de vista internacional. Según la ONU la cultura de paz puede ser caracterizada como un conjunto de valores que están relacionados con el respeto a todos los derechos humanos, el fin de la violencia y la promoción del desarrollo. Esa Declaración de la ONU cuenta asimismo con un programa de acción con objetivos, estrategias y agentes principales para que se desarrolle ese cambio de una cultura de guerra para una cultura de paz en el mundo.

Las dos guerras mundiales y el fin de la guerra fría fueron determinantes para que organizaciones internacionales - UNESCO y ONU - propusiesen esas Declaraciones y además para que demostrasen la existencia de una gran preocupación por las condiciones de vida actuales.

Sin embargo, es fundamental decir que la paz no significa la ausencia de conflictos como comúnmente se suele pensar. Por lo tanto, es importante traer el concepto de paz imperfecta propuesto por Muñoz (2004); en ese planteamiento, la paz es percibida como algo que convive juntamente con la idea de conflictos. En esa concepción, se presupone que los conflictos son inherentes à actividad humana, o sea, donde hay seres humanos hay conflictos porque las personas tienen intereses y proyectos diferentes. 
Dentro de la historia de la paz, Muñoz y Martínez (2004, p. 50-51) van a explicar lo que entienden como paz imperfecta:

[...] todas aquellas experiencias y estancias en las que los conflictos se han regulado pacíficamente (firma de tratos, paz silenciosa, solidaridad, cooperación, actividades de baja entropía, etc.). [...] Como vemos, no se trata de paces absolutas y perfectas, sino más bien de actos regulativos, transformadores y cotidianos de los conflictos. Son, más bien formas de paz imperfecta porque no es una paz total, ni está absolutamente presente en todos los espacios sociales, sino que convive con la violencia interpretando y tratando de regular el conflicto por la vía pacífica.

Consecuentemente, la idea de conflictividad necesariamente está presente en la noción de paz imperfecta y es comprendida en el contexto de las relaciones entre actores humanos (individuo, grupo, institución, nación, etc.) que tienen planes y propósitos distintos. Esa es la base del conflicto. La diferencia es que esos conflictos pueden ser, en muchos ocasiones, gestionados pacíficamente. Y eso es una cualidad de la paz imperfecta. Así, los conflictos existen y pueden ser solucionados sin el uso de la fuerza o la violencia. Pensar en la paz imperfecta implica pensar en el arreglo de los conflictos, de acuerdo con la multiplicidad de características y circunstancias involucradas en el conflicto, o sea, para gestionar un conflicto de modo pacífico es necesario considerar las interacciones entre los diversos actores, las causas, los diversos intereses y las percepciones presentes.

La idea de paz imperfecta puede ser traducida como "aquellas instancias en las que se puede detectar acciones que crean paz y las interacciones entre ellas, a pesar de que estén en contextos en los que existen conflictos y la violencia y, por lo tanto, convivan con ellos” (MOLINA RUEDA; MUÑOZ, 2010, p. 51).

De ese modo, la búsqueda de la paz imperfecta es siempre por el equilibrio dinámico entre los diversos actores, elementos e interacciones. Ese equilibrio puede ser definido, a partir de conceptos elaborados por Maturana (2000) que describió los seres vivos como seres determinados por sus estructuras, pero con una alta capacidad de transformación a través de las interacciones con el medio ambiente y con otros seres vivos.

Eso significa que, al largo de una vida, un ser vivo posee infinitas interacciones que cambian su dinámica y son responsables por tornarlo lo que es hoy. Y que será un ser vivo distinto el día siguiente porque otras interacciones ocurrirán e irán transformándolo nuevamente. El punto central es que un ser vivo es el resultado de su historia de interacciones con otros y con el entorno. El individuo, a partir de su estructura y posibilidad, incorpora los cambios y también altera el medio ambiente y los otros seres con quién interacciona. Ese funcionamiento de incorporar los cambios puede ser llamado de equilibrio dinámico. 
Otro elemento importante para Maturana es que todo ser vivo y también todo sistema social pasan necesariamente por dos procesos que pueden ser denominados como conservación o variación a lo largo de su existencia. Es decir que todas las interacciones pueden resultar en conservación o mantenimiento del ser vivo o en diferenciación de lo que era antes, por lo tanto, sufren cambios en su dinámica.

Además y de acuerdo con este autor, es posible hacer una analogía entre el nivel individual y micro, y el nivel macro en el que otros actores también siguen esa trayectoria de transformación a partir de las interacciones ocurridas. Se supone que lo que pasa a un sujeto puede ser transpuesto para dimensiones mayores, como, por ejemplo, una institución o una nación. La diferencia es que, en este caso, los diversos niveles de interacción tendrán que ser evaluados para comprender el conflicto y gestionar de forma pacífica los problemas identificados.

En consecuencia, el equilibrio dinámico es un mecanismo central para que los seres humanos podamos mantener las condiciones de nuestra existencia, lo que incluye las relaciones con el entorno y a su vez, las interconexiones de éstas con las relaciones entre los propios seres humanos. Podemos resaltar, como un ejemplo importante, el papel que juega la homeostasis, ya citada anteriormente, como una cualidad autorregulativa compartida con el resto de los seres vivos, que busca el equilibrio y que, en cierto sentido, podría tener sus correspondencias con la cooperación y la búsqueda de la armonía (MOLINA RUEDA; MUÑOZ, 2010, p. 53).

Es importante tener en cuenta también que la idea de paz imperfecta tiene un enfoque transdisciplinar, ya que dialoga con muchos campos teóricos distintos. En cuanto la orientación multidisciplinar trabaja con la idea de agregar varias disciplinas diferentes y la interdisciplinar significa la interacción entre diversas disciplinas, la transdisciplinariedad admite la creación de nuevos objetos y visiones de mundo. Igualmente, la transdisciplinariedad posee una dirección más abierta, en comparación con las otras dos orientaciones, porque transciende los campos clásicos de las ciencias y trabaja también con otras perspectivas, como, por ejemplo, las artes y las experiencias subjetivas.

Según Passos y Barros (2000, p. 74), la noción multidisciplinar puede ser entendida como un "movimiento de disciplinas que se suman en la tarea de aprehender un objeto, que por su naturaleza multifacética, exigirían distintas miradas". Desde esa definición, es posible comprender la perspectiva multidisciplinar como la junción de diferentes disciplinas para pensar un determinado objeto. Ya la propuesta interdisciplinar, de acuerdo con los mismos autores, presupone "la creación de una zona de intersección entre ellas (disciplinas) donde un objeto sería designado" (PASSOS; BARROS, p. 74). Ello significa que en la orientación interdisciplinar hay la proposición de un nuevo espacio donde distintas disciplinas dialogan respecto a un objeto específico. Sin embargo, la crítica hecha por los autores por encima citados es que, en ambas nociones, las fronteras disciplinares (de los objetos y de los sujetos de los saberes) están mantenidas, no habiendo cambios significativos. 
Sin embargo, la perspectiva transdisciplinar trabaja con la idea de subversión de las fronteras del conocimiento, tanto del objeto como de los investigadores. Es decir, hay un cuestionamiento de la clásica dicotomía sujeto-objeto, demostrando que los saberes son heterogéneos, dinámicos y mutables. Ellos así comprenden la noción transdisciplinar:

Pues no solo el objeto es construido, sino que el sujeto y el
sistema teórico o conceptual con los cuales se identifican,
ellos también son efecto emergente de un plan de constitución
en lo cual no se puede tener la unidad y la homogeneidad
de una disciplina o campo científico, ya que (el plan de
constitución) es compuesto de materiales heteróclitos, de
distintos géneros (PASSOS; BARROS, 2000, p. 76). ${ }^{1}$

En ese sentido, el enfoque transdisciplinar combina con la noción de paz imperfecta, ya que para pensar la paz, hace falta considerar diferentes actores, grupos, interacciones, conflictos y dinámicas. Como son elementos y componentes de distintas disciplinas, no sería posible comprender la conexión entre ellos sin tener en cuenta la perspectiva transdisciplinar.

Otro concepto que suele ayudar en la comprensión de la paz imperfecta es la idea de complejidad. Trayendo Morin (2011, p. 35) como el principal pensador de esa teoría, es posible definir la noción de complejidad como algo que:

[...] comprende incertidumbres, indeterminaciones, fenómenos aleatorios. La complejidad, en un cierto sentido, siempre posee relación con el acaso. Así que la complejidad coincide con una parte de incertidumbre, sea proveniente de los límites de nuestro entendimiento, sea inscrita en los fenómenos. Pero la complejidad no se reduce a la incertidumbre, es la incertidumbre en el seno de sistemas ricamente organizados. ${ }^{2}$

De ese modo, hay que pensar la paz imperfecta necesariamente como un sistema transdisciplinar y también complejo, pues se trata de un campo que contiene distintas áreas del conocimiento, además de caracterizarse como un espacio dinámico de interacciones, niveles y actores.

Luego, para comprender cualquier fenómeno - desde el punto de vista transdisciplinar y de la complejidad - no es posible utilizar la linealidad común del método científico, haciendo necesario la ampliación de la visión que se tiene del fenómeno. Por ejemplo, el enfoque de la sociología, por sí solo, no es suficiente para alcanzar el entendimiento de determinados conflictos. Es primordial tener también la opinión de otras disciplinas e instancias involucradas.

Asimismo, es necesario reconocer que, a pesar de la gran cantidad de informaciones, existe una limitación del ser humano que es fundamental en la idea de complejidad. Así como la paz es imperfecta, también lo es el ser humano y la 
tarea principal es aceptar esa imperfección. Significa decir que no es posible tener todo el conocimiento sobre algo, hay una imposibilidad inherente que forma parte de esa idea de complejidad.

De acuerdo con la UNESCO (2010), en su Cátedra Itinerante "Edgar Morin" para el Pensamiento Complejo, la complejidad puede ser definida como:

Complejidad significa aquí, la emergencia de procesos, hechos u objetos multidimensionales, multirreferenciales, interactivos (retroactivos y recursivos) y con componentes de aleatoriedad, azar e indeterminación, que conforman en su aprehensión grados irreductibles de incertidumbre. Por lo tanto un fenómeno complejo exige de parte del sujeto una estrategia de pensamiento, a la vez reflexiva, no reductiva, polifónica y no totalitaria/totalizante. Un contexto inédito $\mathrm{y}$ enorme requiere un pensamiento creativo, radical y polifónico. Un pensamiento exorbitante (capaz de pensar fuera de la órbita de los lugares comunes).

Aún en esa propuesta de complejidad, hay otro componente de extrema relevancia que es el giro epistemológico que ocurre cuando se piensa en el concepto de paz imperfecta. Hacer el giro epistemológico presupone superar la idea de linealidad científica, o sea que es imprescindible tener en cuenta que la ciencia no es capaz de explicar los fenómenos humanos y de la naturaleza como se tuviesen una determinada orden. El paradigma científico hegemónico del Occidente ha instituido la creencia de que existe una orden en los acontecimientos que puede ser descifrada con investigaciones científicas. Morin (2011) suele llamar ese tipo de paradigma como "simplificador", pues él tiende a explicar hechos complejos como se fuesen sencillos. Esa visión de mundo empezó con Descartes, con la separación entre sujeto y objeto, filosofía y ciencia, por lo tanto, subjetividad y objetividad.

En realidad, la teoría de la complejidad va a comprender los eventos como integrantes de sistemas organizados que poseen orden, desorden y multiplicidad. Uno de los principales cambios introducidos por Morin fue la noción de desorden que está presente en los acontecimientos, juntamente con la idea de multiplicidad. Según el autor (2002[1977], p. 99):

El universo no es solamente construido a pesar del desorden, él es construido en y por la desorden, es decir, en y por la catástrofe original y las rupturas que se siguieron, en y por el desdoblamiento desordenado del calor, en y por las turbulencias, en y por las desigualdades de procesos que comandaron toda materialización, toda diversificación, toda interacción, toda organización. El desorden está en acción por toda parte. Él permite fluctuaciones, alimenta la constitución y el desarrollo de fenómenos organizados. Todo devenir está marcado por desordenes: rupturas, cismas, desvíos, nacimientos. [...] Todo desorden no es un entidad per se, él es siempre relativo a procesos energéticos, interactivos, transformadores o dispersivos. ${ }^{3}$ 
La perspectiva compleja convida a pensar la realidad como un campo donde la orden y el desorden conviven dinámicamente y hacen parte de sistemas organizados más amplios. Considerando el paradigma cartesiano que aún predomina en diversos ámbitos de la construcción del conocimiento científico, es difícil hacer ese giro epistemológico. Por lo tanto, hace falta deconstruir conocimientos previos, creencias y valores que están acomodados en el sentido común y reconocer que la realidad y los fenómenos explicados de acuerdo con el paradigma cartesiano son simplificadores.

Lo mismo sucede con el concepto de paz, ya que, al menos en el mundo occidental, la paz está comúnmente asociada a una ausencia de violencia. Por lo tanto, se piensa más en términos de violencia y problemas que en términos de paz y soluciones pacíficas de los conflictos. La propuesta de la paz imperfecta es justamente hacer la junción de los dos elementos: paz y conflictos, comprendiéndolos como parte de una misma dinámica. Por lo tanto, es inevitable hacer ese cambio de visión y tener una nueva mirada acerca de los fenómenos relacionados a los conflictos y la violencia en el mundo para comprender el campo transdisciplinar de la paz.

\section{El CONCEPTO DE PODER PARA LA PAZ IMPERFECTA}

Hecho ese preámbulo que relaciona la idea de paz imperfecta, sus cualidades y los conflictos, hay que entender como se integra el poder en esa dinámica de relaciones e interacciones.

Primeramente, es necesario reflexionar el lugar del poder en las relaciones humanas, o sea, entender que el poder ocupa un espacio relevante en las dinámicas sociales. Aquí no se considera solamente el poder del Estado o del gobierno, como se suele pensar en las ciencias sociales o políticas. Lo interesante no es pensar el poder en cuanto una cosa per se y que pertenece a algo o alguien en particular, sino considerar el poder como un ejercicio constante y presente en todas las interacciones humanas. Significa decir que es posible vislumbrar el poder en las relaciones entre sujetos y no de modo estático en lugares o autoridades.

Según Martínez (2004, p. 360), para algunos autores, el poder es una cosa que se muestra a través de sus efectos, como la gravedad o la electricidad; por lo tanto, sería más fácil describir sus resultados que identificar su naturaleza y fundamento. Ello significa que explicar la idea de poder no es una tarea sencilla, ya que existen innúmeras formas de manifestación del poder. Al mismo tiempo, el poder está distribuido en la sociedad de distintas maneras, sea en instancias y/o interacciones, haciendo que cualquier tentativa de definición suene incompleta o imprecisa. Es decir que el poder está en las personas, grupos e instituciones y sobre todo en todas las interacciones entre seres humanos. Esa afirmación parece obvia, entre tanto el poder está frecuentemente asociado al uso de la violencia y en el Estado, a través de las fuerzas policiales, militares y los partidos políticos. Considerar el poder solamente como el poder violento y/o el poder de ámbito exclusivamente estatal distorsiona la realidad e impide una reflexión adecuada 
sobre el tema. En verdad, el poder es fundamentalmente una capacidad que puede ser desarrollada o no por todos los individuos en sus más distintas interacciones. El poder, en la noción de paz imperfecta, está así descrito:

[...] el poder tendría como característica una calidad de circular y transformarse, de estar en muchas partes, de cambiar de manos, de modificarse constantemente, lo que hace más inestable e inseguro el "poder instrumental" [...]. De manera que si admitimos que poder es potencia y posibilidades, en consecuencia, si cualquiera logra encauzar, desplegar y controlar esas características en el sentido de hacer que sean energías creativas y no simple fuerza ciega, bruta y violenta, sino como poder justificado y proyectivo, todo ello significa que la gente ejerce el principio del autogobierno, extendiendo eso que llamamos nuestra libertad. Todo poder, por tanto, entendido así expresa la acción de nuestra libertad y tiende a liberarnos. Esa forma de ejercicio sería el que, básicamente, busca el poder pacifista (MARTÍNEZ, 2004, p. 361-362).

El texto precedente pone en evidencia nuevas perspectivas de pensar y comprender el poder, pues no está de acuerdo con la orientación tradicional de que él es algo que está ubicado en lugares y/o en personas específicas. En esa concepción, el poder circula y es dinámico, además de poseer distintas formas y niveles de expresión. Sin embargo, la idea de poder, en la paz imperfecta, necesariamente tiene que ser considerada en consonancia con la noción de libertad. Sin esa condición y ese derecho, la posibilidad de ejercer el poder queda disminuida.

Esas reflexiones muestran una nueva mirada al poder porque presentan una perspectiva positiva y creadora del poder, al revés del poder violento; o sea, hay una dimensión de potencia y potencialidad en el poder que generalmente es subestimada. Ello significa también que el poder ostenta facetas variadas, pudiendo caracterizarse como un poder destructivo y/o un poder constructivo, a depender del contexto y actores involucrados.

\section{El concepto de poder para Michel Foucault}

En relación a Michel Foucault, la propuesta no es traer su vasta producción académica, ni hacer un resumen de su obra, sino abordar solamente uno de los varios conceptos que atraviesan su elaboración teórica. El punto central de este artículo es hacer una correlación entre la idea de poder que está presente en la paz imperfecta y en el pensamiento de Foucault y presentar algunas semejanzas existentes entre esas propuestas.

Mientras muchas comprensiones apuntan Foucault como un intelectual que analiza el poder solamente desde las instituciones y del Estado, hay otras lecturas posibles de su obra. Lo importante es considerar que en sus estudios principales - la locura, la sexualidad, el presidio, la justicia - Foucault siempre ha tenido el tema del poder como transversal y fundamental en sus reflexiones. 
Según Pogrebinschi (2004), la gran producción de Foucault ha generado una dispersión y una fragmentación de sus ideas, incluso equívocos en la interpretación y en la comprensión de su obra, ya que el filósofo nunca ha creado una teoría específica sobre el poder, sino que varios análisis sobre el poder desde diferentes ángulos. Aunque Foucault tenga producido diversas cosas sobre el poder y sobretodo la perspectiva del biopoder y del poder disciplinar, ${ }^{4}$ lo importante es plantear un nuevo enfoque relativo al poder.

Para iniciar esa discusión sobre la idea de poder y después presentar las semejanzas con el poder en las investigaciones para la paz, es importante tener en cuenta la respuesta que el propio Foucault (2002) ha dado acerca de cómo ve el poder, en una conferencia que está transcrita en su libro La verdad y las formas jurídicas.

No quise absolutamente identificar poder y opresión. ¿Por qué? Primero porque pienso que no hay un poder, pero que dentro de una sociedad existen relaciones de poder extraordinariamente numerosas, múltiples, en diferentes niveles, donde unas se apoyan en otras y donde unas contestan las otras. [...] Estas relaciones son tan múltiples que no podrían ser definidas como opresión, resumiendo todo en una frase: El poder oprime. No es verdad. El poder no oprime por dos razones: primero, porque da placer, al menos para algunas personas. Tenemos toda una economía libidinal del placer, toda una erótica del poder, ello viene probar que el poder no es sólo opresivo. En segundo lugar, el poder puede crear (FOUCAULT, 2002, p. 153-154).

Así que la visión foucaultiana de poder no necesariamente está relacionada a opresión y tampoco se puede pensar en solamente un tipo de poder. Al mismo tiempo, el autor dice expresamente que el poder es una fuente de placer y también de creación. En ese sentido, la concepción foucaultiana está vinculada a una perspectiva más ampliada, donde el filósofo reconoce la vía creativa del poder y no sólo la visión destructiva y violenta. Deleuze (2005, p. 37), otro gran filósofo y amigo personal de Foucault escribió un libro sobre su obra y añadió relevantes contribuciones:

Pero el poder no tiene esencia, él es operatorio. No es atributo, sino que relación: la relación de poder es un conjunto de fuerzas que pasa tanto por las fuerzas dominadas como por las dominantes, ambas constituyendo singularidades.

Es posible observar que el poder para Foucault también no está en algo o en alguien, como ya mencionado antes en la idea de poder para la paz imperfecta; es básicamente en las relaciones humanas que el poder se expresa. Así que es permitido decir que el poder no posee ninguna propiedad o atributos que lo definan con una concepción fija, sino que es necesario concebirlo como operatorio. Ello significa que cotidianamente el poder entra en acción y produce 
efectos en las interacciones; por lo tanto, no es viable considerarlo como algo que posee una esencial primordial. El mismo autor sigue su reflexión acerca del pensamiento foucaultiano:

[...] él (poder) es menos una propiedad que una estrategia, y sus efectos no son atribuibles a una apropiación, "sino que a disposiciones, a maniobras, tácticas, técnicas, funcionamientos"; "él se ejerce más do que se posee, no es un privilegio adquirido o conservado de la clase dominante, pero el efecto de conjunto de sus posiciones estratégicas". Ese es un nuevo funcionalismo, ese análisis funcional ciertamente no niega la existencia de las clases y de sus luchas, pero as insiere en un cuadro completamente diferente, con otros paisajes, otros personajes, otros procedimientos, diferentes de eses con los cuales se acostumbró la historia tradicional (DELEUZE, 2005, p. 35).

Deleuze hace una especie de traducción de la idea de poder foucaultiano, a partir de ejemplos fornecidos por el propio Foucault; o sea que el poder puede ser visto como un tipo de estrategia, al mismo tiempo en que no es posible pensar en posesión del poder, ya que él se manifiesta en maniobras y tácticas pero no se posee. Por esas razones y características que el poder foucaultiano asume, cabe la conclusión que hay una dimensión de fluidez, circularidad y dinamismo en el modo como el poder se presenta y se exterioriza.

\section{El poder para la paz imperfecta y para Foucault: relaCiones, PRODUCCIONES Y COMPLEJIDADES}

Después de presentar las concepciones de poder para la paz imperfecta y para Foucault, así como sus características principales, se torna factible reflexionar acerca de las semejanzas y/o proximidades existentes entre las dos perspectivas.

El primero hecho que merece ser comentado tiene relación con la descripción de cómo el poder es comprendido en ambos enfoques teóricos, o sea que él no puede ser poseído por algo o alguien, él solamente se ejerce en las relaciones y tampoco puede ser considerado como un atributo sino como relación. Es decir, el poder tiene que ser necesariamente calificado como relación de poder y ese es un punto común entre las dos nociones.

Eso muestra una primera semejanza entre las dos perspectivas y al mismo tiempo evidencia una idea que está distante de la concepción tradicional de poder sólo como algo del Estado, y de las fuerzas coercitivas.

La segunda aproximación entre la idea de poder para la paz imperfecta y el poder que maneja Foucault en su obra radica en la visión del poder como algo fundamentalmente no violento y represivo, sino como la expresión de algo productivo y que produce realidades. 
[...] las grandes teses de Foucault acerca del poder se desarrollan en tres rúbricas: el poder no es esencialmente represivo (ya que "incita, suscita, produce"); él se ejerce antes de poseerse (ya que sólo se posea bajo una forma determinable - clase - y determinada - Estado); pasa por los dominados tanto cuanto por los dominantes (ya que pasa por todas las fuerzas en relación) (DELEUZE, 2005, p. 79).

Ese punto de vista permite presumir que se el poder está en cualquier relación humana, hay una dimensión liberadora y emancipadora al no considerar más el poder sólo como poder del soberano. ${ }^{5}$ El poder posee distintas facetas, conteniendo también tipos de poder capaces de crear y construir, sobre todo, la capacidad de producir hechos, cosas y realidades.

La tercera semejanza existente entre el poder para la paz imperfecta y para Foucault es que la lógica de pensamiento desarrollada por el filósofo tampoco sigue la tradición científica común de linealidad. Para decirlo de otro modo, eso significa que la idea de poder para Foucault (1992) persigue una perspectiva compleja, como se puede constatar en la siguiente afirmación de su autoría cuando él describe algunas precauciones que se debe tener al pensar en el tema del poder:

[...] precaución de método: no considerar el poder como un fenómeno de dominación masiva y homogénea de un individuo sobre los otros, de un grupo sobre los otros, de una clase sobre las otras; sino tener bien presente que el poder, si no se lo contempla desde demasiado lejos, no es algo dividido entre los que poseen, los que detentan exclusivamente y los que no tienen y lo soportan. El poder tiene que ser analizado como algo que circula, o más bien, como algo que no funciona sino en cadena. No está nunca localizado aquí o allí, no está nunca en las manos de algunos, no es un atributo como la riqueza o un bien. El poder funciona, se ejercita a través de una organización reticular. Y en sus redes no sólo circulan los individuos, sino que además están siempre en situación de sufrir o de ejercitar ese poder, no son nunca el blanco inerte o consintiente del poder ni son siempre los elementos de conexión. En otros términos, el poder transita transversalmente, no está quieto en los individuos (FOUCAULT, 1992, p. 152).

Aquí parece haber una propuesta de pensar el poder, a partir de la complejidad, ya que se coloca la circularidad, la forma reticular y la necesidad de verlo como algo transversal que atraviesa las relaciones humanas. En otras palabras, Foucault sugiere pensar el poder fuera de una orientación linear y clásica, desde el punto de vista científico, así como la noción de paz imperfecta. Hay un cambio epistemológico donde hace falta mirar el poder desde otro punto. Teniendo en cuenta las características de la complejidad formuladas por Morin (2002 [1977]), 
uno de los hechos fundamentales es considerar que la complejidad está en la base y no se trata solamente de un atributo o propiedad, sino que una perspectiva de visión distinta. Para ejemplificar, es interesante esa afirmativa:

El sencillo es solo un momento arbitrario de abstracción tirado
de las complejidades, un instrumento eficaz de manipulación
laminando un complejo. La génesis es compleja. La partícula
es compleja. La organización es compleja. La evolución es
compleja (MORIN, 2002, p. 456). ${ }^{6}$

En ese sentido, tanto la orientación foucaultiana como la concepción de la paz imperfecta están inseridas en una perspectiva epistemológica donde la realidad es concebida como compleja y no engloba más orientaciones cartesianas simplificadoras. Por lo tanto, el poder es visto y entendido como parte de una realidad compleja donde hay circularidad, conexiones, orden y desorden.

Los tres aspectos mencionados anteriormente como semejanzas y/o aproximaciones entre el poder para la paz imperfecta y la noción del poder foucaultiana comprueban que hay más elementos comunes do que se suponía, aproximando las dos perspectivas, más do que alejándolas.

Para ambas visiones, el poder es algo que está en todas las relaciones humanas, o sea, no es una propiedad de alguien, sino que una estrategia; no tiene esencia per se, y se manifiesta en diversos niveles y dimensiones. Además, es algo circular y transversal. Otro elemento fundamental es que el poder no necesariamente es represor, sino que puede ser liberador y emancipador.

De modo general, diría que la prohibición, el rechazo, lejos de ser las formas esenciales que adopta el poder, no son sino sus límites extremos. Las relaciones de poder son por encima de todo productivas (FOUCAULT, 2008, p. 166).

Parece imprescindible tener en cuenta que la idea de que el poder es esencialmente relación de fuerza entre individuos, grupos, instituciones y naciones y que ese juego no está relacionado solamente a cuestiones de violencia y prohibiciones. Aunque la idea de fuerza siga casi siempre asociada al uso de la violencia, es necesario vislumbrar también el poder como concepto abstracto que trabaja con la perspectiva de potencialidad. La violencia es tan solo el poder en su condición límite y no su regla. Así, es posible analizar el poder como juego de fuerzas, potencialidades y productividades.

Resumidamente es posible decir que el poder está en todas las relaciones humanas y esas interacciones pueden ser entendidas como productoras de realidades. Al mismo tiempo, esos juegos de poder circulan y transitan en diferentes niveles, construyendo esquemas y desplazamientos, exigiendo una importante capacidad analítica. 
Por fin, ese análisis plantea una orientación distinta do que se suele pensar como es el poder para Foucault y además propone una visión de sociedad dentro de la perspectiva compleja. Por lo tanto, evidencia un modo de organización social no determinista, dinámico, multi-referenciado y con infinitas posibilidades de cambio.

\section{Notas}

${ }^{1}$ Traducción libre de la autora.

${ }^{2}$ Subrayado del original y traducción libre de la autora.

${ }^{3}$ Traducción libre de la autora.

${ }^{4}$ Para más informaciones sobre eses dos conceptos ver: Pogrebinschi (2004).

${ }^{5}$ Foucault profundiza sus estudios acerca del poder en la Arqueología del saber y La verdad y las formas jurídicas cuando crea el concepto de poder-saber, asociado a la producción del conocimiento, de la verdad y del saber.

${ }^{6}$ Traducción libre de la autora.

\section{REFERENCIAS}

DELEUZE, G. Foucault. São Paulo: Brasiliense, 2005.

FOUCAULT, M. Microfisica del poder. 3 ed. Madrid: Endymión, 1992.

FOUCAULT, M. A verdade e as formas jurídicas. 3 ed. Rio de Janeiro: NAU, 2002.

FOUCAULT, M. Un diálogo sobre el poder y otras conversaciones. Madrid: Alianza, 2008.

MARTÍNEZ, M. L. Poder, política y noviolencia. In: MOLINA RUEDA, B.; MUÑOZ, F. A. (Ed.). Manual de Paz y Conflictos. Granada: Universidad de Granada, 2004. p. 359-383.

MATURANA, H. Da biologia à psicologia. Porto Alegre: Artmed, 2000.

MOLINA RUEDA, B.; MUÑOZ, F. A. Una Cultura de Paz compleja y conflictiva: la búsqueda de equilibrios dinámicos. Revista de Paz y Conflictos, [S.1.], n. 3, p. 41-66, 2010.

MORIN, E. O método 1: A natureza da natureza (1977). Porto Alegre: Sulina, 2002.

MORIN, E. Introdução ao pensamento complexo. 4 ed. Porto Alegre: Sulina, 2011.

MUÑOZ, F. A. La paz. In: MOLINA RUEDA, B.; MUÑOZ, F. A. (Ed.). Manual de Paz y Conflictos. Granada: Universidad de Granada, 2004. p. 23-41.

MUÑOZ, F. A.; MARTÍNEZ, M. L. Historia de la paz. In: MOLINA RUEDA, 
B.; MUÑOZ, F. A. (Ed.). Manual de Paz y Conflictos. Granada: Universidad de Granada, 2004. p. 45-65.

ONU. Declaración y Programa de Acción sobre una Cultura de Paz. Asamblea General de las Naciones Unidas, 6 de octubre de 1999. Disponible en: <http:// www.fund-culturadepaz.org/spa/DOCUMENTOS/DECLARACIONES, $\% 20$ RESOLUCIONES/Declaracion_CulturadPaz.pdf>. Acceso en: 22 nov. 2010.

PASSOS, E.; BARROS, R. B. A construção do plano da clínica e o conceito de transdisciplinaridade. Psicologia: Teoria e Pesquisa. Brasília, v. 16, n. 1, p. 7179, jan./abr. 2000.

POGREBINSCHI, T. Foucault, para além do poder disciplinar e do biopoder. Lua Nova: Revista de Cultura e Política, São Paulo, n. 63, p. 179-201, 2004.

UNESCO. El Derecho Humano a la Paz: Declaración del Director General de la UNESCO. Paris: Unidad Cultura de Paz (CPP), 1997. Disponible en: $<$ http:// www.unesco.org/cpp/sp/declaraciones/HRtoPeace.htm>. Acceso en: 22 nov. 2010 .

UNESCO. Cátedra Itinerante "Edgar Morin" para el Pensamiento Complejo. Disponible en: <http://www.ciuem.org/?q=node/2>. Acceso en: 22 noviembre 2010.

Recebido em: 21 de abril de 2011

Aceito em: 07 de março de 2012 